\title{
Expression analysis of transglutaminase 2 in premalignant lesions of the cervix
}

\author{
Karen Olivia Bazzo Goulart ${ }^{1,2^{*}}$, Alessandra Eifler Guerra Godoy ${ }^{3,4}$, Isnard Elman Litvin ${ }^{3}$ \\ and Fábio Firmbach Pasqualotto ${ }^{3}$
}

\begin{abstract}
Background: The medical management of low-grade squamous intraepithelial lesions (LSIL) is variable, thus a biomarker could assist with the clinical conduct. Type 2 transglutaminase (TG2) has been proposed as a cellular-interfering factor in HPV infection and carcinogenesis. Therefore, this study has the objective of evaluating TG2 expression in LSIL and highgrade squamous intraepithelial lesions (HSIL) and of relating it to the different HPV viral types.

Methods: This study included 146 patients with suspected LSIL or HSIL detected in routine conventional Papanicolaou tests. The presence of HPV DNA and viral typing was defined by the polymerase chain reaction method (PCR). TG2 Immunohistochemistry (IHC) was conducted according to the manufacturer's instructions; $\mathrm{HC}$ was carried out in an Autosteiner-Link 48 Dako equipment. IHC quantitation was performed by relative expression and by using the software Image J. Qualitative variables, such as frequencies and proportions, were compared by using the $x 2$ test for independent samples. For comparison of the qualitative to the quantitative data, nonparametric Mann-Whitney test was used.

Results: The association between histopathological examination and TG2 was statistically significant $(p<0.05)$. Results showed that patients with normal cervical histopathology and LSIL are locally associated with TG2 expression levels $>50 \%$ $(p<0.05)$, and patients with HSIL are associated with no TG2 expression $(p<0.05)$. The analysis of the samples with the Image J software shows a significant $(p<0,001)$ decrease in TG2 immunostaining in HSIL if compared to normal and to LSIL samples. This demonstrates a correlation between the relative quantification and the results provided by Image J. Analysis of HPV types showed a significant association with HPV11 $(p=0.031)$. This indicates that patients with HPV type 11 had higher TG2 values than patients with different types. Image J analysis showed no significant association between TG2 and HPV viral types.
\end{abstract}

Conclusion: The present data suggest that TG 2 has a high expression in LSIL and normal tissues, and decreased in HSIL. We also observed that its expression is associated with HPV11.

Keywords: Human Papillomavirus, Uterine Cervical Neoplasms, Transglutaminase 2

\section{Background}

Cervical cancer is the fourth most common cancer among women, accounting for the death of 265,000 women each year worldwide [1]. HPV prevalence ranges from $13.7 \%$ to $54.3 \%$ according to the studied population and geographic area in Brazil [2]. It is characterized by uncontrolled replication of the organ epithelium, which compromises the underlying tissue (stroma) and may invade adjacent or distant structures and organs. This type

\footnotetext{
* Correspondence: karenbazzo@gmail.com

${ }^{1}$ Postgraduate in Biotechnology, University of Caxias do Sul (UCS), Francisco Getúlio Vargas, 1130, 95070-560 Caxias do Sul, RS, Brazil

${ }^{2}$ Health Sciences Center, University center of Serra Gaúcha, Caxias do Sul, RS, Brazil Full list of author information is available at the end of the article
}

of cancer is related to human papillomavirus (HPV), which is responsible for the appearance of cancer precursor lesions. The mechanism involved in the appearance of cancer is related to a persistent infection by a large number of HPV types, which depends on the virus type and site of infection, as well as on host factors that regulate the virus persistence, regression, and latency [3-5].

Several proteins are important in the HPV infection process. Two HPV proteins are relevant to the cervical cancer mechanism. One of them is E7, which is associated with $\mathrm{pRb}$ (retinoblastoma tumor suppressor protein) and other members of the protein family. This protein disrupts the association between the pRb and E2 factor family. The 
other protein is E6, associated with p53, which prevents growth arrest or apoptosis in response to E7-mediated cell cycle entry in the upper epithelial layers, which might otherwise occur through the activation of the ADPribosylation factor pathway [6].

Type 2 transglutaminase (TG2) has been proposed as a cellular-interfering factor in HPV infections [3, 7]. TG2 belongs to the transglutaminase (TG) iso-enzyme family, which is related to multiple functions [8]. TG2 participates in signal transduction by activating and hydrolyzing the guanidine triphosphate (GTP) enzyme. In normal tissues, TG2-GTP binding is proportional to the TG2 expression level. Furthermore, TG2 acts in the regulation of the extracellular matrix (ECM) stability and in the reconstruction of the intracellular cytoskeleton. Interestingly, TG2 is also an active participant in promoting mobility of malignant cells, and in inducing chemo-resistance to cancer cells [9].

A small number of studies have shown a relationship between HPV infection and TG2 activity and expression. Apparently, TG2 catalytic activity can incorporate polyamine into HPV18 E7, thereby inhibiting its binding to pRb. Moreover, TG2 can translocate from cytosol into nucleus incorporating polyamines into the retinoblastoma protein $(\mathrm{Rb})$ to protect it from caspase-mediated degradation, suggesting that TG2 interacts with $\mathrm{pRB}$ both in the cytosol and in the nucleus [10-13].

Currently, medical management of low-grade squamous intraepithelial lesions (LSIL) is variable $[14,15]$. Biomarkers could contribute to evidence indicators of more serious injuries or to their resolution. Therefore, this study aims to evaluate TG2 expression in low and high-grade precursor lesions and relate it to different viral HPV types.

\section{Methods}

\section{Patient and tissue samples}

This retrospective study has been approved by the Review Board at Faculdade Fátima on the advice number 183.756 and all patients have given written informed consent. Women seen at the Lower Genital Tract Pathology Service of the Central Outpatient Clinic (UCS) and at the Diagnosis Laboratory were included in this study. This outpatient clinic is a secondary referral unit for women sent by primary health care units in the region due to Pap smear abnormal results.

The study included patients with abnormal conventional Pap smear and subsequent cervical biopsy under colposcopic guidance on suspicion of viral infection, changed colposcopic examination or molecular biology examination positive for HPV DNA.

Cervicovaginal samples were obtained from all patients and frozen at $-20^{\circ} \mathrm{C}$ in TE buffer for molecular biology studies. Subsequently, based on a flowchart prepared by the outpatient department of the Lower Genital Tract
Pathology service, patients were submitted to a colposcopy and to a guided biopsy if a lesion was found.

A transversal analysis of 146 formalin-fixed cervical biopsies obtained from women who underwent biopsy between 2003 and 2008 was performed. The study included patients sexually active, non-pregnant patients who were all but negative for other sexually transmitted diseases. The women were aged between 20 and 50 years old (average age of 35 years old).

\section{Cytopathological and histopathological analysis}

Histopathological analyses were processed by the paraffin embedding technique with $3 \mu \mathrm{m}$ thick histological sections, using staining with hematoxylin-eosin (HE), and a subsequent analysis by a pathologist using an optical microscope [16].

Classification was oriented as follows: negative when no significant cytological changes were observed; LSIL when cellular changes consisted of mild dysplasia or CIN 1 and HPV infection were present; HSIL when cellular changes consisted of moderate dysplasia (CIN 2) or severe dysplasia (CIN 3). The control group was composed of samples negative for LSIL or HSIL, all patients in control group were evaluated, including for multiple levels of $\mathrm{H} \& \mathrm{E}$, in order to exclude focal squamous intraepithelial lesions.

\section{HPV typing}

Cervical-vaginal swabs for HPV-DNA detection by the PCR method were collected at the time of the gynecological examination. The determination of HPV DNA presence and viral typing was performed by the polymerase chain reaction method (PCR) [17]. The DNA samples obtained from cervical smears were isolated and purified by using GFX Genomic DNA Purification Kit Blood ${ }^{\circledR}$ kit (Amersham Biosciences, Piscataway, NJ, USA), according to the manufacturer's instructions.

After the DNA extraction, the samples were subjected to PCR using a set of generic primers for HPV, PGMY 09/11 [17], which are capable of amplifying a 450 base pair (bp) from L1 gene of different types of genital HPV. GH20 and PCO4 primers are added to the same PCR. They amplify 268 bp of the human $\beta$-globin gene and serve as internal control to assess the integrity and completeness of DNA from each sample.

The samples were amplified in the first PCR reaction using degenerate primers GP-E6-3F (GGG WGK KAC TGA AAT CGG T), GP-E6-5B (CTG AGC TGT CAR NTA ATT GCT CA) and GP-E6-6B (TCC TCT GAG TYG YCT AAT TGC TC), and W, A/T; K, G/T; R, A/G; $\mathrm{Y}, \mathrm{C} / \mathrm{T}$ and $\mathrm{C}, \mathrm{A} / \mathrm{C} / \mathrm{G} / \mathrm{T}$. These primers amplify E6 and E7 regions of the 38 most common HPV types. Nested PCR reaction was performed specifically with the types $45,11,16,6,18,42,52,33,58,53,39,54,66,51,31,67$, 
59, 62, 69, 35 e 68 . All samples were analyzed by both the first reaction (PCR) and the second reaction (nested PCR). Some patients infected with HPV had the type classified as not defined due to the fact that HPV type was not identified by any specific PCR technique used.

\section{TG2 immunohistochemistry}

Immunohistochemistry (IHC) to TG2 was carried out according to the manufacturer's instructions. IHC was conducted in an automated manner in an AutosteinerLink 48 Dako equipment.

This TG2 IHC assay utilized a monoclonal mouse antibody (Diagnostic Biosystems, Diluition 1:50) directed against the human TG2 domain. The negative control reagent is a monoclonal rabbit IgG isotype control (DA1E; Cell Signaling Technology, Danvers, MA). The deparaffinization, rehydration, and target retrieval were performed by a PT Link (Dako PT100). Slides were then processed by an Autostainer Link 48 (Dako AS480) using an automated staining protocol validated for TG2 IHC assay. IHC staining protocol includes sequential application of a peroxidase-blocking reagent, TG2 primary antibody or negative control reagent, mouse anti-rabbit IgG linker, visualization reagent consisting of secondary antibody molecules and horseradish peroxidase coupled to a polymer backbone, 3,3'-diaminobenzidine tetrahydrochloride (DAB) chromogen reagent with hydrogen peroxide substrate, and a DAB enhancer which modifies the color of precipitated chromogen. Reagents utilized in addition to TG2 assay components included a wash buffer specially formulated for automated IHC staining and a hematoxylin counterstain. IHC-stained slides were mounted in a nonaqueous permanent mounting medium.

TG2 expression by staining patterns is remarkable. Cells showing TG2 expression are shown stained in brown, which could be seen both in the nucleus and in the cytoplasm.

\section{Interpretation and quantification of the staining}

The extent of immunoreactivity in the samples was assessed by two different pathologists, using the same microscope with a 40x objective lens with a field diameter of $0.52 \mathrm{~mm}$. The official histopathology classification of the samples was not disclosed before the scoring was performed.

Analysis of protein expression by immunohistochemistry was performed in two ways. The first immunohistochemical results were evaluated considering the overall proportion of positive cells: samples considered positive for TG2 had their fields photographed. Sections were chosen to give a representative selection of tissue morphologies and stain intensities. Samples were photographed and individually dysplastic and not dysplastic cells were counted, thereby establishing a percentage of positivity.
Cells with both cytoplasmic and nuclear staining were considered to be positive. TG2 relative quantification was categorized into three different intervals markings: $>50 \%$, $\leq 50 \%$ or negative. This classification was based on a previous study [3]. Uncategorized relative expression were also evaluated.

In the second evaluation mode images were scanned and transferred to a computer, and the intensity of staining was defined using $\mathrm{NIH}$ Image J 1.36b software (National Institutes of Health, Maryland, USA). Image J is a public-domain image-analyzing software on Java platform inspired by NIH Image software for Apple's Macintosh. Therefore, it can be run in different operating systems provided they have a suitable Java virtual machine. The repertoire of the software's functions can be extended through different off-the-shelf plug-ins, available on the web (18). NIH Image J 1.36b software was downloaded from their website (http://rsb.info.nih.gov/ij). The color selection and classification of positively stained points was done by using a distribution diagram for the colors red, green and blue (RGB), which shows the changes in intensity and color saturation. This distribution provides information on pixel quantity of the analyzed image. This was obtained through the Threshold Colour plug-in, in which the color range of interest was defined. The software colors the areas that meet this standard white and the remaining areas black [18-20].

Squamous epithelial cells were included in the evaluation and stromal cells were excluded. Initially, the intensity values were grouped into bands of 10 and the corresponding regions in the image were confirmed by using the threshold feature. Thus, initially all intensities from 0 to 10 were turned white on an image with a known pathological score of high positive. Similarly, the zone containing the lightest color shade of pixel intensities was also determined by using an image with a known pathological score of negative. This was because once the highest intensity (high positive) and the lowest intensity (negative) zones were determined, it would help a better determination of the size of the intermediate (positive and low positive) zones. The region between 0 and 60 contained pixels of the high positive stained images. Similarly, samples with known pathological lower scores were used to optimize the correct range.

Immunohistochemical quantification carried out by Image J were compared and evaluated in order to verify the effectiveness and acceptance of methods.

\section{Statistical analysis}

Statistical analysis was performed using SPSS - Statistical Package for Social Sciences (version 20.0). For comparison of qualitative variables as frequencies and proportions, $\chi 2$ test was used for independent samples with a 
possible analysis of adjusted residuals. Ordinal qualitative and quantitative data were compared with nonparametric Mann-Whitney test with the level of significance set at $5 \%$.

The variable HPV was dichotomized into "high risk" and "low risk". According to the literature [21] the following types were considered high-risk HPVs: 16, 18, 31, $33,35,39,45,51,52,56,58,59,26,53,66,67,68,69$, 70,73 and 82 . The low-risk types are classified as 6,11 , $40,42,43,44,55,54$ and 62 . If patients presented any type of high and low risk concomitantly, they were classified as high risk HPV. To evaluate the possible association of TG2 expression with different types of lesions, and to assess the percentage of positivity, relative quantification was categorized into intervals of $\leq 50 \%,>50 \%$ and 0 (negative).

\section{Results}

TG2 and Histopathological analysis of samples for medical pathologist

A total of 146 patients' results were analyzed, 32 patients were excluded due to histological sample with insufficient quality for immunohistochemistry. Thus, 114 out of 146 patients underwent histopathological examination. According to histopathological analysis, the control group consisted of 38 cases (33.3\%), LSIL of 44 cases (38.6\%) and HSIL of 32 cases (28.1\%). When evaluated, uncategorized relative expression of TG2 was observed in $93 \%$ of the samples. In the control group there was an average $74 \%$ positivity. In the LSIL group immunostaining was $74.7 \%$, and in the HSIL group there was an average expression of $22.3 \%$ (Table 1), non-dysplastic cells were also recorded in LSIL and/or HSIL. Significant interobserver variation was not observed.

Association between LSIL, HSIL, control goup and TG2 was statistically significant $(p<0.05)$. Histopathological analysis was performed with $\chi 2$ test complemented by Adjusted Residual Analysis at a significance level of $5 \%$. Results showed that patients with normal samples and LSIL were locally associated with expression levels of TG2 $>50 \%(p<0.05)$, and patients with HSIL were associated with no TG2 expression $(p<0.05)$ (Fig. 1a, b). A significant difference related to HSIL and LSIL relative quantification $(p<0.05)$ was observed.
The analyses of samples by using Image J software show a significant $(p<0.001)$ decrease in immunostaining of TG2 in HSIL if compared to normal and LSIL samples (Fig. 1d), immunostaining ranged from zero to 198.17.. This depicts a correlation between relative analysis by the pathologist and Image $\mathrm{J}$.

When analyzing quantification of Image J, in relation to relative quantification a significant difference $(p<0.001)$ was observed (Fig. 1g, e). This demonstrates a relationship between the two analytical methods (Fig. 1f).

\section{Distribution of HPV in cervical lesions}

All evaluated patients were tested for HPV through PCR, all 146 cases showed amplification of the human $B$ globin gene $(100 \%)$, confirming the adequacy of the specimen regarding the quantity and integrity of the genetic material. Patients were classified as high and low risk depending on the type of HPV identified. Patients who had both low and high risk viral types were classified as high risk. Some patients infected with HPV had the type classified as not defined due to the fact that the HPV type was not identified by any specific PCR technique used.

The evaluated control group consisted of patients without malignant precursor lesions, including cases of squamous metaplasia, endocervicitis and cervicitis. In this group all patients had squamous metaplasia, and 76.3\% of these presented HPV infection. It is important to note that even in the presence of HPV, the collected samples showed no related precursor lesions of high or low degree. Among patients in the control group, 42.1\% were infected with high-risk genotypes, $2.6 \%$ with lowrisk $\mathrm{HPV}, 31.6 \%$ with non-defined types and $23.7 \%$ were negative for HPV.

LSIL cases show the presence of high-risk HPV in $54.5 \%$ of the cases, low-risk HPV in $6.8 \%$, not defined HPV in $29.5 \%$ and negative in $30.1 \%$. HSIL cases showed the presence of high-risk HPV in $50 \%$ of the cases, lowrisk HPV in 9.4\%, not defined HPV in $28.1 \%$, and negative HPV in $9.4 \%$. These results demonstrate the highest prevalence of high-risk genotypes in LSIL (Table 1).

\section{TG2 and HPV typing}

Regarding the diagnosis of HPV infection, the presence of the virus was observed in 124 cases (84.9\%), among

Table 1 Description of sample groups and its relation to TG2 expression

\begin{tabular}{|c|c|c|c|c|c|c|c|c|c|c|c|c|}
\hline \multicolumn{13}{|l|}{ HPV typing } \\
\hline \multirow{2}{*}{$\begin{array}{l}\text { Histopathological } \\
\text { analysis }\end{array}$} & \multicolumn{2}{|c|}{ Patients } & \multirow[b]{2}{*}{$\mathrm{n}$} & \multirow[b]{2}{*}{$\%$} & \multicolumn{2}{|c|}{ Low risk } & \multicolumn{2}{|c|}{ High risk } & \multicolumn{2}{|c|}{ Not defined } & \multirow[t]{2}{*}{ TG2 (\%) } & \multirow[t]{2}{*}{ TG2 - Image J } \\
\hline & $n$ & $\%$ & & & $n$ & $\%$ & $n$ & $\%$ & $n$ & $\%$ & & \\
\hline Control & 38 & 26.0 & 9 & 23.7 & 1 & 2.6 & 16 & 42.1 & 12 & 31.6 & $74 \% *\left(S D^{*} 17.13\right)$ & 110,9 (SD 87.27) \\
\hline LSIL & 44 & 30.1 & 4 & 9.1 & 3 & 6.8 & 24 & 54.5 & 13 & 29.5 & $74,7 \% *$ (SD 17.55) & 80,4 (SD 66.03) \\
\hline HSIL & 32 & 21.9 & 3 & 9.4 & 3 & 9.4 & 17 & 53.1 & 9 & 28.1 & $22,3 \% *$ (SD 24.12) & $5,1^{* *}(S D$ 11.67) \\
\hline
\end{tabular}

${ }^{*} p<0.05,{ }^{* *} p<0.001, \mathrm{SD}=$ Standard Deviation, $\% 100$ cell count 

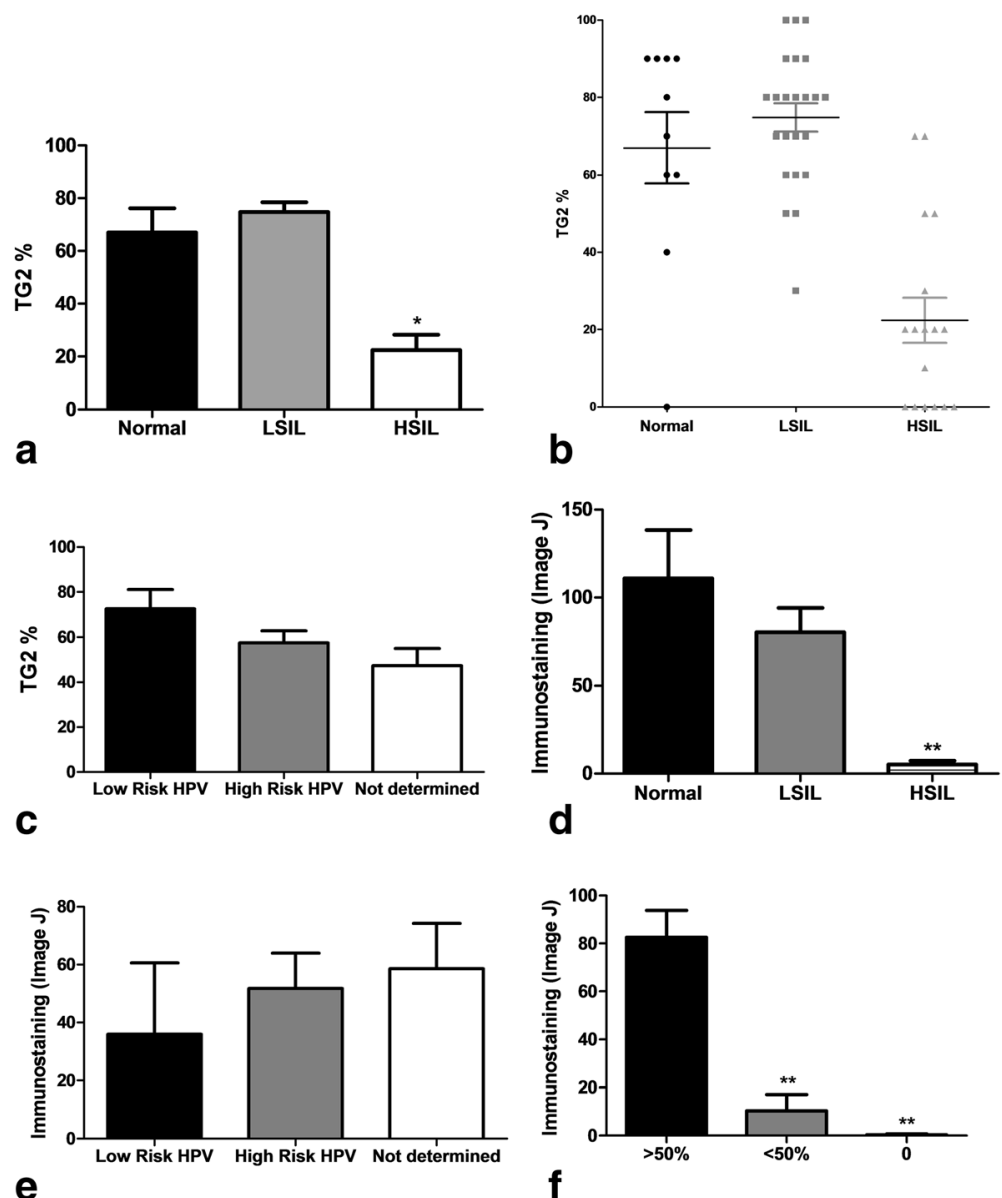

Fig. 1 Distribution of the expression levels of type 2 transglutaminase (TG2) according to different lesion grades (a and b). Distribution of expression levels of type 2 transglutaminase (TG2) according to HPV type (c). Quantification of TG2 by Image J and its association with histopathological classification (d) and viral HPV types (e). Relation between categorization of relative quantification and quantification of TG2 by Image $J(F){ }^{*} p<0,05{ }^{* *} p<0,001$

these 66 patients $(45.2 \%)$ were classified as high risk HPV, 15 (10.3\%) low risk and 43 (29.5\%) with a not defined type of HPV. A total of 22 patients who did not have HPV infection were excluded (Table 2).

TG2 expression was observed in all groups, with an average of $72.5 \%$ for low-risk HPV types, $59 \%$ for highrisk types, and $60.7 \%$ for not defined types (Fig. 1c).

There was no significant statistical association between the relative quantification of TG2 and different classifications of HPV viral types.

A significant similarity between the expression of not defined types and high-risk types can be observed, thus, demonstrating the possible presence of high-risk HPV in the not defined group.

The most frequently identified virus types were 16 with 29 cases, 6 with 20 cases, 11 with 18 cases, and 41 cases belonged to the not defined group. When individual HPV types were analyzed in relation to TG2 expression levels a statistical significance $(p=0.031)$ was demonstrated for
HPV 11 indicating that patients with type 11 had higher values of TG2 if compared to patients with a different type. These results may represent a trend as the evaluation is based on a limited number of patients.

When analyzing TG2 expression according to HPV type, one notices that generally the expression decreases in high-risk types and increases in low-risk (Fig. 1c). Particularly, when types of HPV which are not defined are observed by relative quantification and

Table 2 Description of sample groups and the relation to TG2 expression, data from 124 HPV positive patients

\begin{tabular}{|c|c|c|c|c|c|c|}
\hline \multirow[t]{2}{*}{ HPV typing } & \multicolumn{2}{|c|}{ Patients } & \multicolumn{2}{|c|}{ TG2 (\%) } & \multicolumn{2}{|c|}{ TG2 Image J } \\
\hline & $\mathrm{n}$ & $\%$ & $\%$ & $S D^{a}$ & $\mathrm{n}$ & $S D^{a}$ \\
\hline Low-Risk HPV & 15 & 10.3 & 72.5 & 17.08 & 35,99 & 55.11 \\
\hline High-Risk HPV & 66 & 45.2 & 59 & 31.27 & 51,77 & 72.24 \\
\hline Not Defined HPV & 43 & 29.5 & 60,7 & 29.85 & 58,55 & 70.26 \\
\hline
\end{tabular}

${ }^{a}$ Standard Deviation, \%100 cell count, $S D=$ Standard Deviation 
Image J, TG2 behaves similarly to the high-risk types. This shows the importance of analyzing other viral types, since it is more likely they become malignant tissues (Fig. 1e).

Viral types investigated in this study were defined according to their prevalence in cervical lesions. High and low risk are the most common types identified, however, considering the obtained results, it became relevant to delineate the types which are not defined, owing to the fact that probably among them there are prevalent highrisk viral types. When the association between TG2 and viral HPV types was verified through Image J analysis, no significant association was found (Fig. 1d).

Results suggest that TG2 involving the presence of LSIL and non-malignant alterations (Fig. 2) of the cervix are significantly associated to low-risk viral types. Therefore, TG2 variable expression is associated with cancerous changes of the cervix.

\section{Discussion}

Our data showed a HPV frequency of $84.9 \%$, which corroborates with previously published studies. In this study, samples were obtained from patients with suspected LSIL or HSIL detected in a routine conventional Pap test in clinical pathology of cervical biopsy under colposcopic guidance on suspicion of viral infection, changed colposcopic examination or molecular biology examination positive for HPV DNA. This procedure explains the high prevalence of HPV in the samples.

Among the most common HPV types, genotypes 16, $18,31,33,45,52$ and 58 are responsible for approximately $90 \%$ of cervical cancers [22]. In Brazil, Oliveira et. al. demonstrated that the most frequent types were HPV16 (77.6\%), HPV18 (12.3\%), HPV31 (8.8\%), HPV33 (7.1\%) and HPV35 (5.9\%) [23]. According to other studies, HPV16 is the most prevalent type in all regions of Brazil. HPV 18 is the second most common in North, South, and Southeast of Brazil. Types 31 and 33 are the next most prevalent in the Northeast and central areas of Brazil, respectively [24]. Our data corroborates the information present in the literature, since HPV16 was the most prevalent type in patients, with 29 (19.9\%) positive cases.

The presence of HPV in normal samples is also shown in other studies $[2,22,23]$. In a study performed in the city of Porto Alegre, the prevalence of HPV was demonstrated in $11.6 \%$ of women without LSIL or HSIL [25]. This fact was also shown in this study where HPV infection was found in control samples (76.3\%), which consisted of patients with squamous metaplasia. All patients were evaluated, including multiple levels of $\mathrm{H} \& \mathrm{E}$, to exclude focal squamous intraepithelial lesion.

Our study did not investigate the presence of TG2 in patients with biopsies without any alteration or HPV infection owing to the difficulty of obtaining biopsies for healthy patients. Biopsies of normal tissue were not evaluated in the present study, but TG2 expression in histologically normal squamous and columnar epithelium could be assessed in some samples in adjacent areas to metaplastic, reactive and dysplastic epithelium. Characterization of TG2 expression in normal cervix is seen in only two studies which investigated TG2 levels in samples such as these, even though this is relevant information. Both studies found negative expression in normal tissues, indicating that TG2 expression is solely related to non-malignant changes or LSIL $[3,7]$.

In this study we have analyzed TG2 expression in the epithelium of human cervix in relation to HPV-induced dysplastic modifications. Our findings indicate that TG2 expression levels in patients with metaplasia $(74 \%)$ were similar to patients with LSIL (74.7\%). Moreover, TG2 expression is significantly related to genotype $11(p<0.05)$, which is classified as low-risk. Thus, the correlation of TG2 to productive HPV infections and low malignant potential lesions was observed. Furthermore, results showed that TG2 is less detected in HSIL, whereas it is strongly up-regulated in early cervical lesions with HPV infection.

TG2 has been identified as an interfering factor in HPV infections. This protein is involved in a wide range of functions, such as cell death, cytoskeletal rearrangement and extracellular stabilization. Its modified activation has been associated with a wide range of pathologies [8, 26-28].

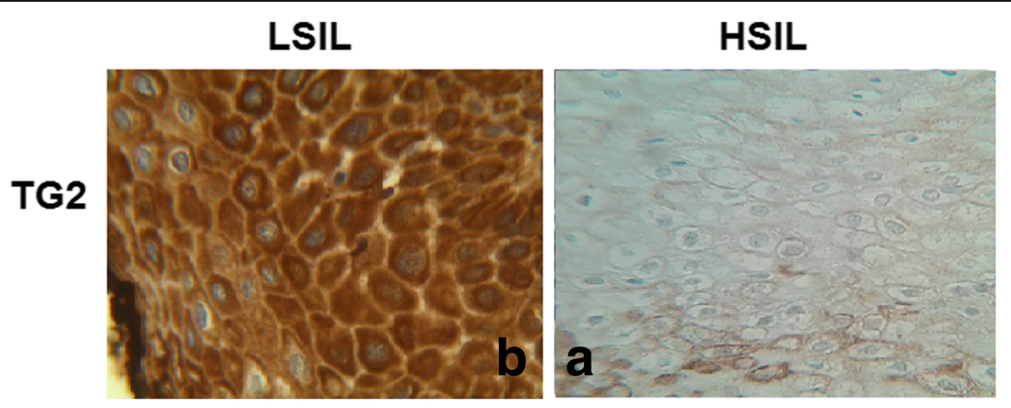

Fig. 2 Transglutaminase type 2 (TG2) immunostaining in high- (a) and low- (b) grade cervical lesions 
TG2 is also associated with viral pathogenesis. Several proteins involved in the process of infection are modified by TG2 [10, 12]. Some studies indicated that TG2 with its catalytic activity can incorporate polyamine in HPV18 E7 protein, thus inhibiting its binding to $\mathrm{pRb}$ protein in the cytosol and nucleus, thereby protecting the caspases degradation pathway $[13,29,30]$. In addition, recently, studies have shown that there is an increase in the splicing process of TG2 in dysplastic cells, indicating a relation between these TG2 molecules and the ones with intracellular cancer alterations [31].

Our results show that LSIL presented high levels of the enzyme, while in HSIL these levels are decreased, which can be related to the absence of HPV 11 in this samples. Moreover, this enzyme is currently referred as a phenomenon not restricted to high-risk genotypes [3]. Present results corroborate previously published data [3, 7], emphasizing that HSIL patients demonstrated a significant statistical association with low TG2 expression $(p<0.05)$.

When the relationship between TG2 and HPV types was analyzed, a significant statistical association to HPV $11(p=0.031)$ was shown. Whereas these viral types are classified as low-risk, data suggest that TG2 can act as an analytical factor in elucidating the development of cervical cancer particularly associated with HPV11. This is evidenced for the first time in the literature.

Currently, HPV types 6 and 11, which are responsible for $90 \%$ of all genital warts, are included in the quadrivalent vaccine for HPV. The infection time of low-risk HPV types is lower if compared to high-risk types. Lowgrade types generate productive lesions, where certain viral gene products stimulate cell proliferation, resulting in benign lesions such as warts. These HPV types do not integrate their genome into cellular DNA [32].

Findings in this study demonstrated for the first time the use of Image J software for quantification of TG2 in cervix samples. Results suggest that the two quantification methods can be applied and corroborate previously published data. In this sense, individual variation is a significant limitation to the relative quantification. This factor could be decisive in some cases and the problem could be overcome by using Image J. This software has been widely used in researches and presents several advantages, such as being a quantitative method, presenting a lower chance of variation in the outcome.

Immunohistochemical staining is a commonly used method for assessment of protein expression in situ. However, it is highly subjective and prone to variation based on both experience and training and possibly due to unconscious subjectivity. Other authors have demonstrated the application of computational methods in the final interpretation of biopsy slides of cancerous tissue $[33,34]$. Image $J$ software has been used by some authors [25], however, to this day, there are no studies that evolve using Image J software in biopsies of cervical cancer precursor lesions. In the present study, we compared the quantification of slides of cervical biopsies immunostained for TG2 by using Image J, and comparing the results to the relative quantification method.

Based on the data obtained in this study, we concluded that quantification of TG2 in cervical biopsies by using Image $J$ has significant results for improving diagnostic techniques of cervical cancer and therefore might help determining an effective protein expression without inter- or intra-personal variations. Correlation between the quantification by the proposed technique and viral types indicates its practical and reliable applicability.

Further study of HPV-related carcinogenesis processes could generate new controlling strategies, providing a more rational approach to diagnosis and treatment of cervical lesions. Understanding that LSIL lesions are not always true cervical cancer precursors has raised expectant management of women with these lesions. However, management approaches are still in disadvantage by the incapacity to better predict who is at risk for HSIL and cancer and who is not; particularly in LSIL treatment [15].

Moreover, the treatment decision of LSIL depends on a number of complex factors related to the patient. New biomarkers that could predict which LSIL are at a highest risk for development of HSIL appear to be on prospect and may make the management of LSIL as clear as present guidelines for HSIL [15]. In the present study we evidenced the behavior of an important biomarker in HPV carcinogenesis process. TG2 showed a differential expression in LSIL and HSIL, which can be an indication that this enzyme behaves as a marker.

TG2 decreases its expression in HSIL cases, which can be related to absence of HPV 11 in these samples. TG2 expression in association with other lesion markers is not widely investigated. In a paper in which TG2 was correlated with p16INK4a, a regulatory protein of the cell cycle and widely studied in cervical cancer, an inverse correlation was observed between the markers (Pearson: -0.930) [3]. Another research, however, did not show any correlation between the expression of both markers [7]. Nevertheless, the association between TG2 and other biomarkers should be investigated in order to optimize the use of this tool associated with other proteins related to cervical cancer, thereby raising its analytical power.

\section{Conclusion}

Our data suggest that TG2 decreases in immunostaining in HSIL if compared to normal and LSIL samples. The analysis of HPV types showed a significant association with HPV11. This indicates that patients with HPV type 11 had higher TG2 values if compared to patients with other types. 


\section{Abbreviations}

HPV: Human papillomavirus; HSIL: High-grade squamous intraepithelial lesion; IHC: Immunohistochemistry; LSIL: Low-grade squamous intraepithelial lesions; PCR: Polymerase chain reaction method; TG2: Type 2 transglutaminase

\section{Acknowledgements}

Coordination for the Improvement of Higher Education Personnel (CAPES). National Counsel of Technological and Scientific Development (CNPQ). University of Caxias do Sul (UCS).

\section{Funding}

Coordination for the Improvement of Higher Education Personnel (CAPES). National Counsel of Technological and Scientific Development (CNPQ).

\section{Availability of data and materials}

All data generated or analyzed during this study are included in this published article.

\section{Authors' contributions}

KB, AEGG. IEL and FFP participated in the study analyzing samples and writing the article. All authors read and approved the final manuscript.

\section{Competing interests}

The authors declare that they have no competing interests.

\section{Consent for publication}

The authors agree to deliver to the responsible editor(s) on a date to be agreed upon the manuscript created according to the Instructions for Authors and consent for publication.

\section{Ethics approval and consent to participate}

This study was approved by the Review Board at Faculdade Fátima on the advice number 183756

\section{Consent to publish statement}

This retrospective study has been approved by the Review Board at Faculdade Fátima on the advice number 183.756 and all patients have given written informed consent.

\section{Publisher's Note}

Springer Nature remains neutral with regard to jurisdictional claims in published maps and institutional affiliations.

\section{Author details}

${ }^{1}$ Postgraduate in Biotechnology, University of Caxias do Sul (UCS), Francisco Getúlio Vargas, 1130, 95070-560 Caxias do Sul, RS, Brazil. ${ }^{2}$ Health Sciences Center, University center of Serra Gaúcha, Caxias do Sul, RS, Brazil. ${ }^{3}$ Medical school of UCS, University of Caxias do Sul (UCS), Francisco Getúlio Vargas, 1130 95070-560 Caxias do Sul, RS, Brazil. ${ }^{4}$ IPCEM - Institute of Clinical Research for Multicentric Studies, Francisco Getúlio Vargas, 1130, 95070-560 Caxias do Sul, RS, Brazil.

Received: 12 July 2016 Accepted: 5 May 2017

Published online: 03 July 2017

\section{References}

1. Ferlay J, et al. Cancer incidence and mortality worldwide: sources, methods and major patterns in GLOBOCAN 2012. Int J Cancer. 2015;136(5):E359-86.

2. Ayres ARG, Silva GA. Cervical HPV infection in Brazil: systematic review. Rev Saude Publica. 2010;44(5):963-74.

3. Del Nonno F, et al. Role and predictive strength of transglutaminase type 2 expression in premalignant lesions of the cervix. Mod Pathol. 2011;24(6):855-65.

4. Wentzensen $\mathrm{N}$, et al. Triage of HPV positive women in cervical cancer screening. J Clin Virol. 2015;28(15):00749.

5. Wieringa $\mathrm{HW}$, et al. Breaking the DNA damage response to improve cervical cancer treatment. Cancer Treat Rev. 2015;24(15):00227-3.

6. Fiedler $\mathrm{M}$, et al. High level HPV-16 E7 oncoprotein expression correlates with reduced pRb-levels in cervical biopsies. Faseb J. 2004;18(10):1120-2.
7. Gupta R, et al. Tissue transglutaminase 2 as a biomarker of cervical intraepithelial neoplasia (CIN) and its relationship to p16INK4A and nuclear factor kappaB expression. Virchows Arch. 2010;456(1):45-51.

8. Fesus $L$, Piacentini M. Transglutaminase 2: an enigmatic enzyme with diverse functions. Trends Biochem Sci. 2002;27(10):534-9.

9. Begg GE, et al. Mechanism of allosteric regulation of transglutaminase 2 by GTP. Proc Natl Acad Sci U S A. 2006;103(52):19683-8.

10. Amendola A, et al. "Tissue" transglutaminase expression in HIV-infected cells: an enzyme with an antiviral effect? Ann N Y Acad Sci. 2001:946:108-20.

11. Lu W, Strohecker A, Jh JHO. Post-translational modification of the hepatitis $C$ virus core protein by tissue transglutaminase. J Biol Chem. 2001;276(51):47993-9.

12. Jeon JH, Kim IG. Role of protein modifications mediated by transglutaminase 2 in human viral diseases. Front Biosci. 2006;11:221-31.

13. Jeon $\mathrm{JH}$, et al. Transglutaminase 2 inhibits $\mathrm{Rb}$ binding of human papillomavirus E7 by incorporating polyamine. Embo J. 2003;22(19):5273-82.

14. Tranbaloc P. Natural history of precursor lesions of cervical cancer. Gynecol Obstet Fertil. 2008:36(6):650-5.

15. Cox JT. Management of women with cervical cancer precursor lesions. Obstet Gynecol Clin North Am. 2002:29(4):787-816.

16. Solomon, D. and R. Nayar. The Bethesda System for reporting cervical cytology: definitions, criteria, and explanatory notes 2004: Springer Science \& Business Media

17. Gravitt $P$, et al. Improved amplification of genital human papillomaviruses. J Clin Microbiol. 2000;38(1):357-61.

18. Schindelin J, et al. The Image J ecosystem: An open platform for biomedical image analysis. Mol Reprod Dev. 2015;82(7-8):518-29.

19. WU JH, YANG KT. Experiment research of edge detection in digital image processing. J Hunan Inst Sci Technol (Nat Sci). 2007;20(2):25-7.

20. Matos LL, et al. Immunohistochemistry quantification by a digital computer-assisted method compared to semiquantitative analysis. Clinics. 2006;61(5):417-24

21. Souho T, Benlemlih M, Bennani B. Human papillomavirus infection and fertility alteration: a systematic review. PLoS ONE. 2015;10(5):e0126936.

22. Wagner $\mathrm{M}$, et al. Global availability of data on HPV genotypedistribution in cervical, vulvar and vaginal disease and genotype-specific prevalence and incidence of HPV infection in females. Infect Agent Cancer. 2015;10(13):015-0008

23. de Oliveira CM, et al. Human papillomavirus genotypes distribution in 175 invasive cervical cancer cases from Brazil. BMC Cancer. 2013;13(1):357.

24. Rabelo-Santos $\mathrm{S}$, et al. Human papillomavirus prevalence among women with cervical intraepithelial neoplasia III and invasive cervical cancer from Goiânia, Brazil. Mem Inst Oswaldo Cruz. 2003;98(2):181-4.

25. Becker $\mathrm{E}$, et al. Prevalence and epidemiologic correlates of atypical squamous cells of undetermined significance in women at low risk for cervical cancer. Diagn Cytopathol. 2001;24(4):276-82.

26. Falasca $L$, et al. Transglutaminase type $\|$ is a key element in the regulation of the anti-inflammatory response elicited by apoptotic cell engulfment. J Immunol. 2005;174(11):7330-40.

27. Facchiano F, Facchiano A, Facchiano AM. The role of transglutaminase-2 and its substrates in human diseases. Front Biosci. 2006:11:1758-73.

28. Falasca $\mathrm{L}$, et al. Transglutaminase type $\|$ is involved in the pathogenesis of endotoxic shock. J Immunol. 2008:180(4):2616-24.

29. Boehm JE, et al. Tissue transglutaminase protects against apoptosis by modifying the tumor suppressor protein p110 Rb. J Biol Chem. 2002; 277(23):20127-30

30. Milakovic T, et al. Intracellular localization and activity state of tissue transglutaminase differentially impacts cell death. J Biol Chem. 2004 279(10):8715-22.

31. Phatak VM, et al. Expression of transglutaminase-2 isoforms in normal human tissues and cancer cell lines: dysregulation of alternative splicing in cancer. Amino Acids. 2013:44(1):33-44.

32. Minaguchi T, Yoshikawa H. Molecular mechanism of cervical carcinogenesis. Gan To Kagaku Ryoho. 2010:37(1):18-22.

33. Fuchs $T$, et al. Computational pathology analysis of tissue microarrays predicts survival of renal clear cell carcinoma patients. Med Image Comput Comput Assist Interv. 2008;11:1-8.

34. Samsi S, Krishnamurthy AK, Gurcan MN. An efficient computational framework for the analysis of whole slide images: Application to follicular lymphoma immunohistochemistry. J Comput Sci. 2012;3(5):269-79. 